\title{
3D Magnetothermal Simulations of Tangled Crustal Magnetic Field in Central Compact Objects
}

\author{
Andrei P. Igoshev ${ }^{1}$ (D), Konstantinos N. Gourgouliatos ${ }^{2}$ (D), Rainer Hollerbach ${ }^{1}$ (D), and Toby S. Wood ${ }^{3}$ (D) \\ ${ }^{1}$ Department of Applied Mathematics, University of Leeds, LS2 9JT Leeds, UK; a.igoshev@leeds.ac.uk, ignotur@gmail.com \\ ${ }^{2}$ Department of Physics, University of Patras, 26504 Patras, Greece \\ ${ }^{3}$ School of Mathematics, Statistics and Physics, Newcastle University, Newcastle upon Tyne, NE1 7RU, UK \\ Received 2020 December 2; revised 2021 January 19; accepted 2021 January 19; published 2021 March 10
}

\begin{abstract}
Central compact objects (CCOs) are young neutron stars emitting thermal X-rays with bolometric luminosities $L_{\mathrm{X}}$ in the range of $10^{32}-10^{34} \mathrm{erg} \mathrm{s}^{-1}$. Gourgouliatos, Hollerbach, and Igoshev recently suggested that peculiar emission properties of CCOs can be explained by tangled magnetic field configurations formed in a stochastic dynamo during the proto-neutron star stage. In this case the magnetic field consists of multiple small-scale components with negligible contribution of global dipolar field. We study numerically three-dimensional magnetothermal evolution of tangled crustal magnetic fields in neutron stars. We find that all configurations produce complicated surface thermal patterns that consist of multiple small hot regions located at significant separations from each other. The configurations with initial magnetic energy of $(2.5-10) \times 10^{47}$ erg have temperatures of hot regions that reach $\approx 0.2 \mathrm{keV}$, to be compared with the bulk temperature of $\approx 0.1 \mathrm{keV}$ in our simulations with no cooling. A factor of two in temperature is also seen in observations of CCOs. The hot spots produce periodic modulations in light curve with typical amplitudes of $\leqslant 9 \%-11 \%$. Therefore, the tangled magnetic field configuration can explain thermal emission properties of some CCOs.
\end{abstract}

Unified Astronomy Thesaurus concepts: Neutron stars (1108); Magnetic fields (994); Astrophysical magnetism (102); Magnetohydrodynamics (1964); Astrophysical fluid dynamics (101); Magnetohydrodynamical simulations (1966)

\section{Introduction}

Central compact objects (CCOs) are neutron stars (NSs) found in close proximity to the geometric center of supernova remnants (SNRs; Pavlov et al. 2004; de Luca 2008; De Luca 2017). CCOs have soft X-ray luminosities of $10^{32}$ $10^{34} \mathrm{erg} \mathrm{s}^{-1}$, incompatible with cooling luminosities of young NSs with ages of a few kiloyears. Their X-ray spectra generally contain two blackbody components: the first arising from bulk NS thermal emission with temperature $\approx 0.1-0.2 \mathrm{keV}$, and the second produced by a small, thermally emitting area (a few percent of the NS size) with temperatures of $0.2-0.4 \mathrm{keV}$. CCO luminosities are comparable to those of some quiescent magnetars (for a review see, e.g., Kaspi \& Beloborodov 2017). However, CCOs show no activity similar to magnetars (with the exception of 1E 161348-5055 in SNR RCW 103 (Rea et al. 2016), which might be a magnetar with an unusual formation path). There is no indication that they are binaries. CCOs are numerous: up to $30 \%$ of NSs associated with SNRs at distances up to $5 \mathrm{kpc}$ are CCOs (de Luca 2008), which is difficult to reconcile with the Galactic NS birth rate (Keane \& Kramer 2008). It is unclear how CCOs age. CCO-like objects are not found among nearby cooling NSs (Turolla 2009) with ages of $>100$ kyr. Bogdanov et al. (2014) and Luo et al. (2015) searched for radio pulsars that show CCO-like X-ray emission and found none.

Long-term X-ray observations have revealed the spin period and period derivative for three CCOs (Halpern \& Gotthelf 2010; Gotthelf et al. 2013). The spin periods are as follows: RX
J0822-4300 in SNR Puppis A has $P=112 \mathrm{~ms}$ (Gotthelf \& Halpern 2009), CXOU J185238.6+004020 in Kes 79 has $P=105 \mathrm{~ms}$ (Gotthelf et al. 2005), and 1E 1207.4-5209 in PKS $1209-51 / 52$ has $P=424 \mathrm{~ms}$ (Zavlin et al. 2000). From these measurements and their period derivatives, the magnetic dipole inferred for these CCOs can be estimated using the formula $B_{p} \approx 3.2 \times 10^{19} \sqrt{P \dot{P}} \mathrm{G}$ (Lorimer \& Kramer 2004). The inferred values of $B_{p}$ are in the range of $10^{10}-10^{11} \mathrm{G}$, which is one or two orders of magnitude smaller than the $10^{12} \mathrm{G}$ dipole fields of young radio pulsars. The typical spin-down luminosity $\dot{E}$ for CCOs is $\sim 10^{32} \mathrm{erg} \mathrm{s}^{-1}$, which means that magnetospheric currents arising from pulsar spin-down cannot be responsible for formation of compact hot regions at the CCOs' surfaces.

CCOs with measured periods and period derivatives are located among old radio pulsars in the $P-\dot{P}$ plane. While these pulsars are prominent radio sources, CCOs show no nonthermal radio emission, neither persistent nor periodic. Coherent pulsar radio emission is strongly beamed and is generated in the magnetosphere by some kind of plasma instability or, as suggested recently, by nonstationary plasma discharge (Philippov et al. 2020). Even if we miss the beam of radio emission owing to the pulsar orientation, the presence of a pulsar wind nebula might be expected. Young, rapidly rotating NSs embedded in SNRs are often surrounded by a compact pulsar wind nebula (Gaensler \& Slane 2006). This nebula is powered by the particle wind generated by rotating, magnetized NSs. No evidence of pulsar wind nebula is found in relation to CCOs.

Therefore, observational properties of CCOs pose multiple questions for researchers: (1) what the source of additional heating in these NSs is, (2) why their emitting areas are so small, (3) what are the descendants of CCOs, (4) why is no 
radio emission detected, and (5) how different CCOs really are from magnetars. This is a part of a larger problem with a variety of different NS classes. A grand unification of NSs is a scenario that tries to explain the difference between various classes of NSs based on properties and evolution of their magnetic fields (Kaspi 2010; Igoshev et al. 2014). In the framework of this scenario, CCOs might evolve into some other category of NSs, such as radio pulsars at timescales of $10^{4}-10^{6}$ yr.

Several scenarios have been proposed to answer these questions and to explain the origin and evolution of CCOs. These scenarios attribute the additional source of energy to strong hidden magnetic fields of NSs. Among these scenarios are fallback accretion and a stochastic dynamo (Gotthelf \& Halpern 2007). Magnetic fields of NSs are multicomponent; they can include both poloidal and toroidal parts, as well as small-scale fields. Only the large-scale poloidal dipole magnetic field emerging from the surface can be measured using the timing technique.

In the fallback accretion scenario, some material expelled by the supernova explosion does not have enough energy to escape from the system indefinitely, and it is subsequently accreted back onto the NS (Chevalier 1989). This accretion continues after the NS crust has solidified. New infalling material buries the magnetic field, covering it with a new crustal layer (Bernal et al. 2010). The buried magnetic field gradually reemerges through the surface over time (Ho 2011; Viganò \& Pons 2012; Bernal et al. 2013). In this scenario, the excessive heating is explained by the amplification and dissipation of the magnetic field, which is compressed between the superconducting NS core and new crust formed from fallback material. The absence of radio emission is explained by the suppression of the surface dipolar magnetic field, preventing the NS from producing enough plasma in its magnetosphere. The same explanation holds for the lack of a pulsar wind nebula. Igoshev et al. (2016) studied the evolution of small-scale magnetic field after a fallback and found that the field (both small and large scale) reemerges, so these NSs should start operating as normal radio pulsars after $10^{4}-10^{5} \mathrm{yr}$. It is still unclear whether the heat produced by the magnetic field causes the formation of small emitting regions at the surface in this scenario. If a strong fallback occurs at the newly born magnetar, a CCO magnetar could be produced. This is a $\mathrm{CCO}$ that has an extremely strong magnetic field trapped deep in the crust that should occasionally exhibit flares.

In the stochastic dynamo scenario, the NS is born with a predominantly small-scale magnetic field, with only a weak dipole component. This small-scale field is formed during the proto-NS stage, which lasts for tens of seconds (Pons et al. 1999) and ends with the solidification of the crust. During this stage, there are two regions where the matter is unstable according to the Ledoux convection criterion. One of these regions is located around densities of $10^{13} \mathrm{~g} \mathrm{~cm}^{-3}$ of proto-NSs (Thompson \& Murray 2001). Nagakura et al. (2020) analyzed state-of-the-art 3D simulations of supernova explosions for progenitor masses ranging from 9 to $20 M_{\odot}$ and found that convection always develops in proto-NSs. This convection zone is an ideal place for a dynamo that could generate magnetic fields in the range of $10^{12}-10^{15} \mathrm{G}$.

The generation of a large-scale magnetic field is thought to require rapid rotation of the proto-NS (see, e.g., recent simulations by Raynaud et al. 2020). If the proto-NS rotates slowly, a dynamo could still operate, but producing only a strong small-scale field (Thompson \& Murray 2001). Such a field with surface $B_{s} \approx 10^{14} \mathrm{G}$ is hidden for a distant observer, because it hardly contributes to the NS spin-down. A stochastic dynamo could also operate in convective fallback flow (Thompson \& Murray 2001) and also lead to formation of small-scale magnetic fields.

In the stochastic dynamo scenario, the excessive heating of CCOs could be explained by ohmic decay combined with fast Hall evolution of this much stronger nondipolar magnetic field. We test it in our research using the tangled configuration of magnetic field suggested by Gourgouliatos et al. (2020). In these configurations, the magnetic fields are comparable in strength to magnetar ones, but they should not produce similar stresses and therefore should not lead to magnetar-like activity. Gourgouliatos et al. (2020) and Brandenburg (2020) found that these configurations where the poloidal dipole is absent or rather small develop such a component in the course of their evolution owing to an inverse Hall cascade.

The Hall cascade was first suggested for electron-MHD by Goldreich \& Reisenegger (1992) with application to NS crusts. Occurrence of this cascade was confirmed in detailed numerical simulations (see, e.g., Wareing \& Hollerbach 2009, 2010; Brandenburg 2020). Turbulence leads to redistribution of magnetic energy to both small-scale (forward cascade) and large-scale (inverse cascade) fields. In the case of tangled magnetic field configurations, an inverse cascade leads to an increase of the dipolar magnetic field with time. The small dipolar component at the beginning of NS evolution is not sufficient for the activation of the radio-pulsar mode. When the global dipolar component rises after $\sim 10 \mathrm{kyr}$ of evolution, this object might start operating as a radio pulsar, which solves the problem regarding the absence of CCO descendants, because in this case CCOs turn into normal radio pulsars with no unusual properties after a period of time.

This article is structured as follows. In Section 2 we describe the method that we use in our simulations. In Section 3 we present results of three-dimensional simulations and corresponding light curves. We discuss all results and conclude in Sections 4 and 5, respectively.

\section{Methods}

We perform simulations in two steps. First, we compute the surface temperature distribution using an updated version of the MHD code PARODY (Dormy et al. 1998; Wood \& Hollerbach 2015; Gourgouliatos et al. 2016). Next, we compute the light curves and pulsed fraction for different orientations of rotating NSs using the ray-tracing in general relativity.

\subsection{Magnetothermal Evolution}

The details of the magnetothermal code are summarized in De Grandis et al. (2020) and Igoshev et al. (2021). We solve magnetic induction and thermal diffusion equations in the solid crust of NSs using the electron-MHD approximation, in which only electrons carry electric charge and thermal flux. Basically, we solve two coupled partial differential equations. The first is the induction equation:

$$
\begin{aligned}
\frac{\partial \boldsymbol{B}}{\partial t}= & -c \boldsymbol{\nabla} \times\left\{\frac{1}{4 \pi e n_{e}}(\boldsymbol{\nabla} \times \boldsymbol{B}) \times \boldsymbol{B}\right. \\
& \left.+\frac{c}{4 \pi \sigma} \nabla \times \boldsymbol{B}-\frac{1}{e} S_{e} \nabla T\right\},
\end{aligned}
$$


where $\boldsymbol{B}$ is the magnetic field, $n_{e}$ is the electron number density, $e$ is the elementary charge, $c$ is the speed of light, $\sigma$ is the electric conductivity, $S_{e}$ is the electron entropy, and $T$ is the temperature. The second is the heat equation:

$$
\begin{aligned}
C_{V} \frac{\partial T}{\partial t}= & \nabla \cdot(\hat{k} \cdot \nabla T)+\frac{|\nabla \times B|^{2} c^{2}}{16 \pi^{2} \sigma} \\
& +\left(\frac{c}{4 \pi e}\right) T \nabla S_{e} \cdot(\boldsymbol{\nabla} \times \boldsymbol{B}),
\end{aligned}
$$

where $\hat{k}$ is the thermal conductivity tensor. The first two terms in our induction Equation (1) are the ones introduced by Goldreich \& Reisenegger (1992) and represent the Hall evolution and ohmic dissipation, respectively. The third term describes the so-called Biermann battery effect, i.e., magnetic field generation due to temperature gradients (Blandford et al. 1983). This effect is closely related to electron baroclinicity, which acts as a source of electron circulation, and hence magnetic induction.

In the heat Equation (2), the first term represents anisotropic thermal diffusion. While heat flows freely along magnetic field lines, heat transfer is inhibited in the direction orthogonal to field lines. The second term represents heating by ohmic field decay, and the third term represents the entropy carried by electric currents. Unless strong temperature gradients are maintained externally, e.g., by magnetospheric heating (De Grandis et al. 2020), the last terms in Equations (1) and (2) are generally very small in comparison to other terms and play a negligible role in the field evolution.

We write the thermal conductivity tensor in the following form:

$$
\left(\hat{k}^{-1}\right)_{i j}=\frac{3 e^{2}}{\pi^{2} k_{B}^{2} T}\left(\frac{1}{\sigma} \delta_{i j}+\frac{\epsilon_{i j k} B_{k}}{e c n_{e}}\right),
$$

where $\delta_{i j}$ is the Kronecker delta, $\epsilon_{i j k}$ is the Levi-Civita symbol, $k_{B}$ is the Boltzmann constant, and $B_{k}$ is the field component along the $k$-axis.

Electron entropy is related to temperature and chemical potential $\mu(r)$ of a degenerate, relativistic Fermi gas as follows:

$$
S_{e}=\frac{\pi^{2} k_{B}^{2} T}{\mu} .
$$

Similar to Gourgouliatos \& Cumming (2014), we use the following radial profiles for the chemical potential, electron density, and conductivity:

$$
\begin{aligned}
& \mu(r)=\mu_{0}\left(1+\frac{1-r}{0.0463}\right)^{4 / 3} \\
& n_{e}(r)=n_{0}\left(1+\frac{1-r}{0.0463}\right)^{4} \\
& \sigma(r)=\sigma_{0}\left(1+\frac{1-r}{0.0463}\right)^{8 / 3},
\end{aligned}
$$

where $\mu_{0}=2.9 \times 10^{-5} \mathrm{erg}, \quad n_{0}=2.5 \times 10^{34} \mathrm{~cm}^{-3}$, and $\sigma_{0}=1.8 \times 10^{23} \mathrm{~s}^{-1}$. For computational convenience we assume that the heat capacity is proportional to the temperature in the form (see, e.g., Page et al. 2004)

$$
C_{V} \propto \sigma T,
$$

so that Equation (2) becomes a linear equation in $T^{2}$.

\subsection{Boundary and Initial Conditions}

For the magnetic induction at the inner boundary, we assume the Meissner condition, i.e., zero radial component of magnetic field $B_{r}=0$ and zero tangential component of the current $J_{t}=0$ at the crust-core boundary; see De Grandis et al. (2020) and Hollerbach \& Rüdiger (2004) for more details. Physically this corresponds to a scenario in which the field is expelled from the core before the crust solidifies. In reality, the core might still contain some magnetic field, although the timescale on which the core field evolves is uncertain; see, e.g., Gusakov et al. (2020). For the magnetic field at the outer boundary we use vacuum boundary conditions, i.e., we assume that $\boldsymbol{\nabla} \times \boldsymbol{B}=0$ in the region outside the star. This condition is implemented numerically as

$$
\frac{\partial B_{\mathrm{p}, l}^{m}}{\partial r}+\frac{l+1}{r} B_{\mathrm{p}, l}^{m}=0
$$

and

$$
B_{\mathrm{t}, l}^{m}=0
$$

where $B_{\mathrm{p}, l}^{m}$ and $B_{\mathrm{t}, l}^{m}$ represent the coefficients of the poloidal and toroidal magnetic potentials, which are expanded in series of spherical harmonics of degree $l$ and order $m$. Physically these boundary conditions correspond to neglecting any electric currents in the star's tenuous plasma atmosphere. We note that CCOs have no radio emission, and no pulsar wind nebula was ever detected around a $\mathrm{CCO}$, so their magnetospheres might have less plasma than is typical for normal radio pulsars.

For the temperature, at the crust-core interface we assume no cooling, yielding the boundary condition $\partial T / \partial t=0$. In reality, the core cools at a rate determined by neutrino emission, which is practically insensitive to conditions within the crust. Since our focus in this study is on small-scale temperature anomalies produced within the crust, we neglect the core cooling for simplicity. At the outer boundary we assume that the heat flux out of the computational domain is subsequently emitted as blackbody radiation from the star's surface, i.e.,

$$
-\left.\boldsymbol{r} \cdot \hat{\kappa} \cdot \nabla T\right|_{b}=\sigma_{S} T_{s}^{4},
$$

where $\sigma_{S}$ is the Stefan-Boltzmann constant, $T_{b}$ is the temperature at the top of the computational domain, and $T_{s}$ is the effective surface temperature. We assume that these two temperatures are related by the thermal blanket equation

$$
\left(\frac{T_{b}}{10^{8} \mathrm{~K}}\right)=\left(\frac{T_{s}}{10^{6} \mathrm{~K}}\right)^{2},
$$

similar to a relation suggested by Gudmundsson et al. (1983), but in a more simplified form. This assumption is necessary in our calculations because the thermal relaxation timescale of the envelope is much shorter than any other evolution timescale involved in our simulations. As was shown by Gudmundsson et al. (1982), the effective surface temperature depends essentially only on the temperature in deep layers (below densities $10^{10} \mathrm{~g} \mathrm{~cm}^{-3}$ ) and surface gravity.

We use the same magnetic field configurations as in Gourgouliatos et al. (2020), so the initial magnetic field consists of several high-order multipoles with $10 \leqslant l \leqslant 20$. We choose phases randomly for these fields. The amount of initial magnetic energy $E_{\mathrm{tot}, 0}$ in this field is set at the beginning of simulations. A dipolar poloidal magnetic field is added to the 
Table 1

Numerical Models Computed Here

\begin{tabular}{lccccc}
\hline \hline Name & $\begin{array}{c}E_{\text {tot, } 0} \\
(\mathrm{erg})\end{array}$ & $\begin{array}{c}B_{\text {dip, }, 0} \\
(\mathrm{G})\end{array}$ & $\begin{array}{c}\left\langle B_{0}\right\rangle \\
(\mathrm{G})\end{array}$ & $p_{\text {max }, 1}$ & $\begin{array}{c}\mathrm{PF}_{\mathrm{m}} \\
(\%)\end{array}$ \\
\hline Model 1 & $2.5 \times 10^{45}$ & 0 & $2 \times 10^{14}$ & 0.58 & 0.3 \\
Model 2 & $2.5 \times 10^{45}$ & $10^{10}$ & $2 \times 10^{14}$ & 0.58 & 0.3 \\
Model 3 & $2.5 \times 10^{45}$ & $10^{11}$ & $2 \times 10^{14}$ & 0.58 & 0.3 \\
Model 4 & $2.5 \times 10^{47}$ & $10^{10}$ & $2 \times 10^{15}$ & 0.44 & 11 \\
Model 5 & $2.5 \times 10^{47}$ & $10^{12}$ & $2 \times 10^{15}$ & 0.43 & 11 \\
Model 6 & $1 \times 10^{48}$ & $10^{10}$ & $5 \times 10^{15}$ & 0.39 & 9 \\
Model 7 & $1 \times 10^{48}$ & $10^{12}$ & $5 \times 10^{15}$ & 0.39 & 9 \\
\hline
\end{tabular}

Note. $p_{\max , 1}$ is the value of total magnetic energy decay exponent at its first maximum. $\mathrm{PF}_{\mathrm{m}}$ is the maximum pulsed fraction computed for all models at age $3.5 \mathrm{kyr}$.

stochastic magnetic field configuration with fixed $B_{\text {dip, o value }}$ before the simulations are started.

We compute the same first five models as in Gourgouliatos et al. (2020). In models 6 and 7 we decrease the total magnetic energy in the crust by a factor of 4 . We make this change because for larger energies we encounter certain numerical problems. We summarize the details of our simulations in Table 1.

\subsection{Light Curves and Pulsed Fraction}

In order to compute the light curves, we follow the same technique as described in Igoshev et al. (2021). Namely, NS orientation is described using three angles: $i$ is the angle between line of sight and rotational axis of NS, $\kappa$ is the angle between magnetic dipole moment and rotational axis, and $\Delta \Phi$ is the initial phase. These three angles uniquely describe NS orientation at the start of rotation. To simulate rotation of an NS, we use simplified equations presented by Beloborodov (2002) combined with

$$
\begin{aligned}
\mu= & \sin i[\cos \Phi \cos \theta \sin \kappa \\
& -\sin \theta(\sin \phi \sin \Phi-\cos \kappa \cos \phi \cos \Phi)] \\
& +\cos i[\cos \kappa \cos \theta-\cos \phi \sin \kappa \sin \theta],
\end{aligned}
$$

where $\theta$ and $\phi$ are coordinates at the NS surface with respect to original dipolar magnetic poles, $\mu=\cos \psi$ is the direction toward the position with coordinates $\theta, \phi$ at infinity, and $\Phi \in[0,2 \pi]$ is the angular phase. Our Equation (13) reduces to Equation (5) of Beloborodov (2002) if a hot spot is located at the magnetic pole, i.e., $\theta=0=\phi$.

We choose the photon beaming to be proportional to $\cos ^{2} \theta^{\prime}$ (DeDeo et al. 2001) because of the following argument. In an atmosphere that is not affected by magnetic field, the darkening is described by the Hopf function; see, e.g., Chandrasekhar (1950). The intensity emitted at angle $90^{\circ}$ is $\approx 1 / 3$ of the intensity emitted in the normal direction. In a strong magnetic field (van Adelsberg \& Lai 2006) the beaming is stronger than this, so at angles of $80^{\circ}$ the emission is 0.1 of its value at $10^{\circ}$, and it goes to 0 at $90^{\circ}$ (see Figure 15 of van Adelsberg \& Lai 2006), which is roughly similar to $a \cos ^{2} \theta^{\prime}$ behavior. For CCOs the pulsed fractions are typically small, so it is better to overestimate the pulsed fraction in our models to check whether the model has enough predictive power.

The flux emitted by each surface element of NS is integrated over the visible hemisphere (which is slightly more than half of the star owing to the light bending in a strong gravitational field) to produce the visible flux for each rotational phase. We use 16 phases to simulate a light curve. For a few orientations we compute the pulsed fraction $(\mathrm{PF})$ as

$$
P F=\frac{F_{\max }-F_{\min }}{F_{\max }+F_{\min }},
$$

where $F_{\max }$ and $F_{\min }$ are maximum and minimum fluxes for a fixed NS rotational orientation. We select the maximum pulsed fraction among different orientations and show them in Table 1.

In Igoshev et al. (2021), we fitted observed light curves of magnetars in quiescence to estimate the NS rotational orientation. This was possible because magnetars seem to have regular large-scale magnetic fields. This is not the case for CCOs. If their magnetic fields are indeed formed as a result of a stochastic dynamo, the dipolar component is weak and changes location relatively quickly (see Gourgouliatos et al. 2020). Additional loops of magnetic field are located randomly; therefore, it is necessary to describe the location of each individual loop of magnetic field to reproduce a thermal map. Thus, there is no use in exact fitting of the light curve, since multiple slightly different magnetic field configurations will result in very similar light curves but slightly different NS rotational orientations.

\section{Results}

We computed models 1-3 up to $50 \mathrm{kyr}$ and models $4,5,6$, and 7 up to $100 \mathrm{kyr}$. We show the surface temperature distributions for models 2, 4, and 6 in Figures 1-3. The surface temperature distributions in the case of models 1 and 3 are very similar to that of model 2 . The surface temperatures of models 5 and 7 are very similar to ones produced in models 4 and 6 , respectively, so we do not plot these maps. Basically, the surface temperature distributions are defined only by the value of the initial magnetic energy, since the initial field topology is the same in all models. The initial strength of the dipolar component weakly affects the values of surface temperatures and the location of hot and cold regions.

In all cases the small-scale structure of the magnetic field leads to formation of a complicated thermal pattern that includes alternating small hot and cold regions. Individual hot spots have simple shapes, but temperatures of groups of spots differ in different parts of NSs, making the pattern even more complicated. Overall, at $3.5 \mathrm{kyr}$ the surface temperature map is composed of a few relatively small hot regions and hot spot associations and a much more extended colder continuum. We measure the linear sizes of several hot regions in the case of model 6 at $1.2 \mathrm{kyr}$. Two brightest hot regions have linear sizes of 2.2 and $2.6 \mathrm{~km}$, respectively, assuming that the NS radius is $10 \mathrm{~km}$. At later stages of evolution $(>20 \mathrm{kyr})$ the temperature and size of hot regions seen in all models decay. The hot regions become completely isolated and cool down.

The hot regions are much hotter in the case of models 4 and 6 , with larger initial magnetic energy than in model 2 . In the case of model 6, which has the largest magnetic field energy, the hot spots are reaching temperatures of $2.16 \times 10^{6} \mathrm{~K}$, which is $\approx 0.19 \mathrm{keV}$. Gotthelf et al. (2013) mentioned that it is necessary for a successful CCO model to show variations of the temperature by a factor of two over the surface, because this temperature difference is seen in observations of $\mathrm{RX}$ J0822-4300. Our models 4, 5, 6, and 7 show temperature variations of a factor of 1.7-2.2 over the surface. 


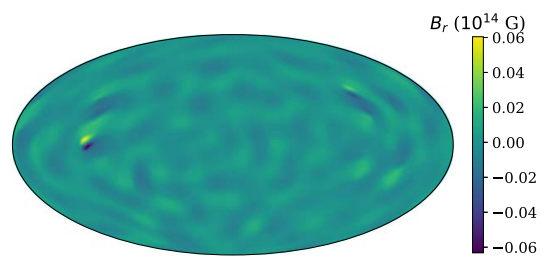

(a)

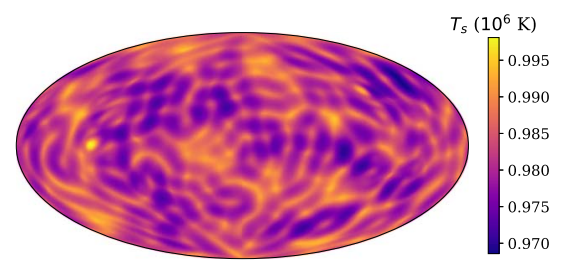

(d)

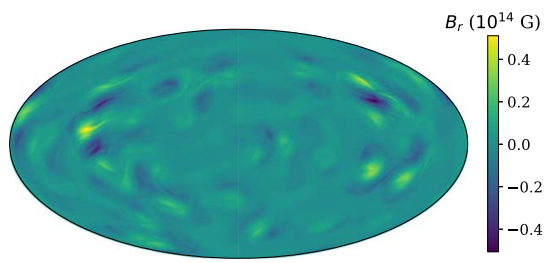

(b)

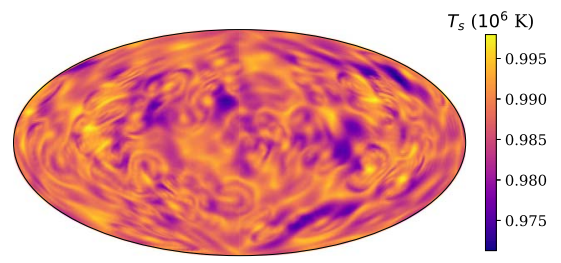

(e)

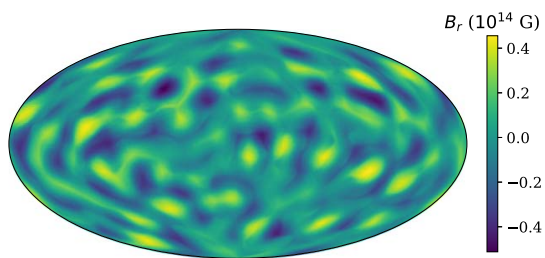

(c)

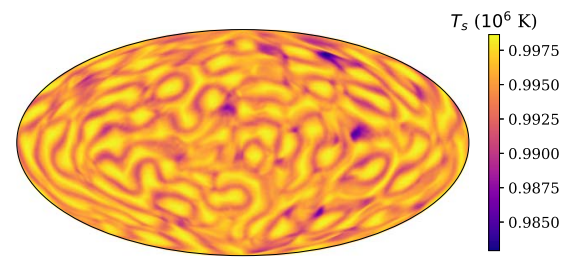

(f)

Figure 1. Surface magnetic fields (top row) and surface temperatures (bottom row) for model 2. Individual panels correspond to different ages: (a, d) 3.5 kyr; (b, e) $9.5 \mathrm{kyr}$; (c, f) $37.7 \mathrm{kyr}$.

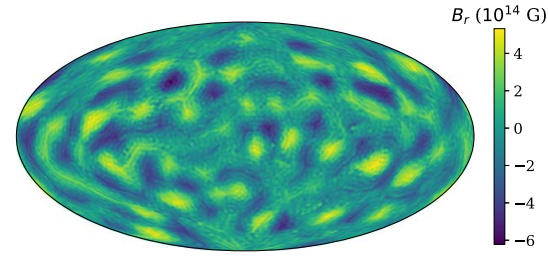

(a)

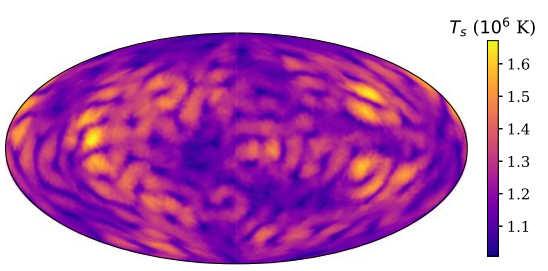

(d)

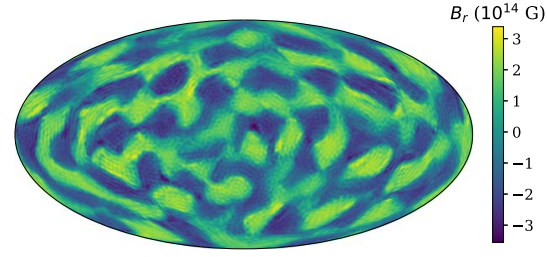

(b)

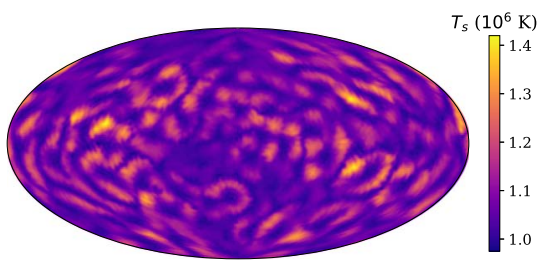

(e)

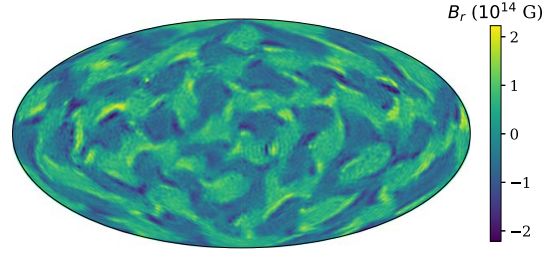

(c)

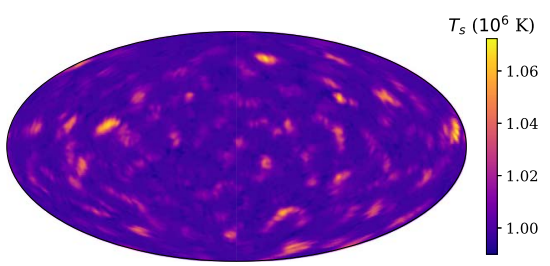

(f)

Figure 2. Surface magnetic fields (top row) and surface temperatures (bottom row) for model 4. Individual panels correspond to different ages: (a, d) 3.5 kyr; (b, e) $9.5 \mathrm{kyr} ;$ (c, f) $37.7 \mathrm{kyr}$.

Surface heating is caused by the release of magnetic energy stored in the crustal field; see Figure 4. During the first $\approx 20 \mathrm{kyr}$, the initial total magnetic energy $E_{\text {tot }}$ decays from $2.5 \times 10^{47}$ erg to $\approx 5 \times 10^{46}$ erg with typical power of $\approx 3 \times 10^{35} \mathrm{erg} \mathrm{s}^{-1}$. In real NSs this power is emitted through the surface photon emission and neutrinos. In our simulations this power is partly emitted through the surface boundary condition and partly absorbed by the inner boundary condition.

We estimate the instantaneous exponent that describes the decay of total magnetic energy,

$$
\mathcal{E}(t)=\int E(k, t) d k,
$$

in a way similar to work by Brandenburg (2020),

$$
p(t)=-\frac{d \log \mathcal{E}(t)}{d \log t} .
$$

We show the results of this evolution in Figure 5. The behavior of $p(t)$ is not monotonic. It reaches the first maximum with values in the range of $0.39-0.58$ (values for individual models can be found in Table 1). This level is reached at different physical times because the Hall timescale differs in these models. After this initial saturation, the $p(t)$ value briefly declines and starts growing again, reaching values of $1-3$ at the end of our simulations; see Figure 5. This means that by the 


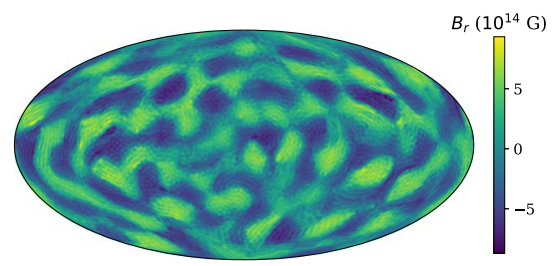

(a)

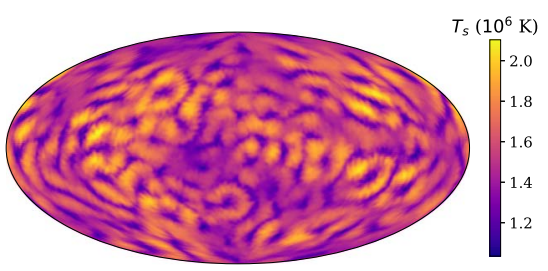

(d)

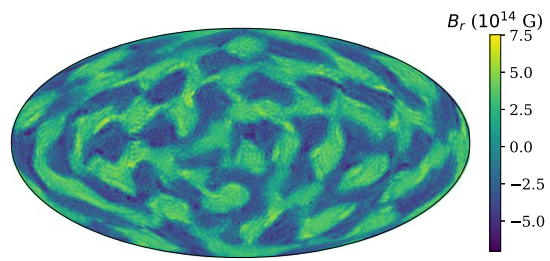

(b)

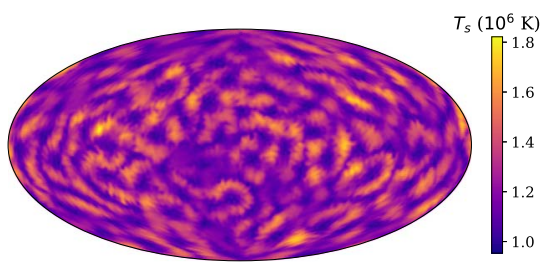

(e)

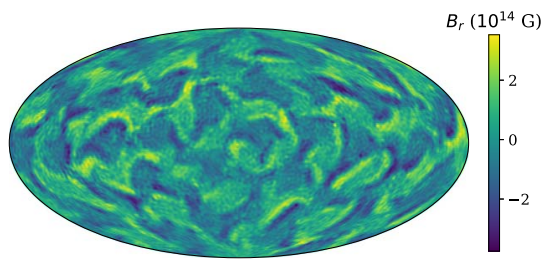

(c)

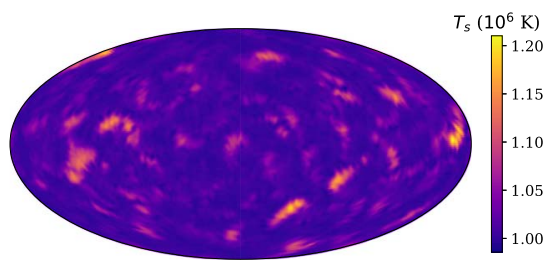

(f)

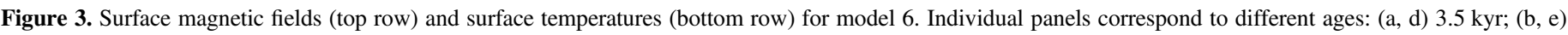
$6.8 \mathrm{kyr}$; (c, f) $38 \mathrm{kyr}$.

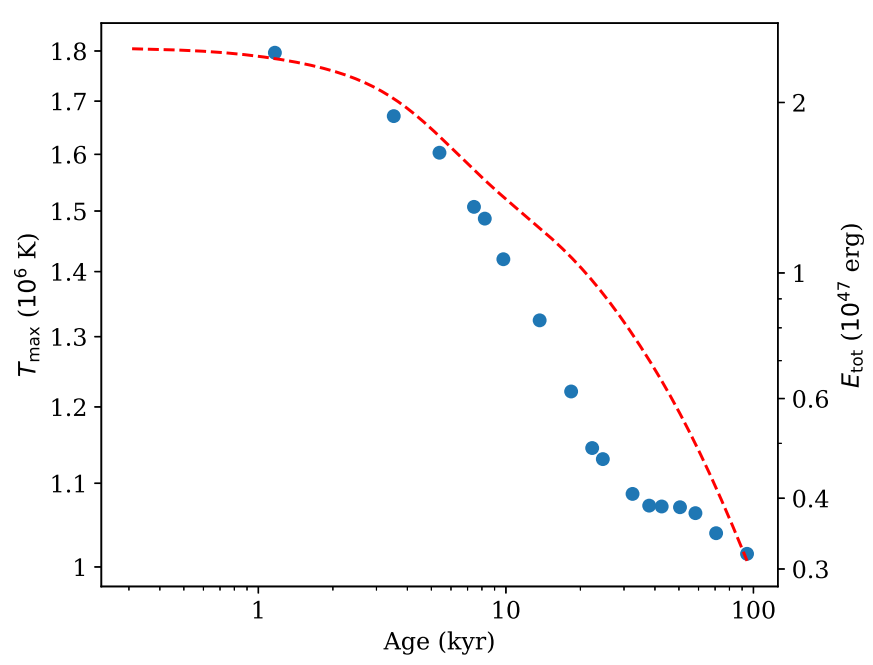

Figure 4. Evolution of maximum surface temperature (blue circles) and total magnetic energy (dashed red line) for model 4.

end of our simulations the energy of magnetic field decays exponentially owing to the ohmic decay.

The values of $0.39-0.58$ correspond to the value $2 / 5$ obtained for the case of helical magnetic fields by Brandenburg (2020). He noticed that even magnetic fields with a small fraction of helicity evolve quickly to nearly $100 \%$ helical configurations. Therefore, it is not surprising that our simulations show an energy decay rate initially of $\mathcal{E}=\mathcal{E}_{0} t^{-2 / 5}$, which is typical for helical configurations.

Another way to describe a complicated field using a single value is by using the rms of magnetic field $B_{\text {rms }}$, similar to what Brandenburg (2020) obtained using the PENCIL code (Brandenburg et al. 2020). We compute $B_{\text {rms }}$ over the whole volume of the NS crust. We show the evolution of the maximum temperature as a function of $B_{\mathrm{rms}}$ in Figure 6. We see that similar values of $B_{\mathrm{rms}}$ might correspond to different maximum temperatures depending on the evolution stage. On the other hand, the value of emerging dipolar, poloidal magnetic field seems to correlate with $B_{\text {rms }}$, as seen in Figure 7. The initial value of the dipolar component gets erased quickly by the Hall evolution, and the field that emerges afterward is related to $B_{\text {rms. }}$. In the case of model 2, the emergent field is $2 \times 10^{10} \mathrm{G}$. In the case of models 6 and 7 , the dipolar field reaches values of $\approx 10^{12} \mathrm{G}$, quite independently of the initial dipolar magnetic field.

It is interesting to note that the Hall evolution creates vortices similar to turbulence seen in the surface temperature maps, especially in Figure 1, age 9.5 kyr. Typically the Hall evolution creates power spectra somewhat similar to turbulence, but with a different slope of $l^{-2}$; see, e.g., Wareing \& Hollerbach (2009) and Goldreich \& Reisenegger (1992). When the Hall evolution starts with an initial condition consisting of a strong global dipole, it mostly preserves the dipole component and redistributes a part of its energy into small-scale magnetic fields. The intensity of these fields is small in comparison to the dipole, and they are not distinguishable as spatial structures; see, e.g., Igoshev et al. (2021). In simulation 2 we suppress the initial dipole and choose the small-scale harmonics to be strong at the beginning of the simulation. As a result, we see that the magnetic energy is redistributed among small-scale harmonics in such a way that spatial vortices emerge in the thermal map, which shows that under certain conditions the Hall turbulence is very similar to regular turbulence. The system evolution of model 2 in scales larger than the crust scale height $H$ is affected by the geometry of the system. This roughly corresponds to modes with $\ell \approx \pi R_{\mathrm{NS}} / H$, with $\ell$ being the spherical harmonic decomposition mode and $R_{\mathrm{NS}}$ the radius of the star. For a value of $H \sim 1 \mathrm{~km}$ this corresponds to modes with $\ell>30$, where the behavior of the turbulence will be more profound and the field will not be affected by the geometry of the system.

Since the hot regions occur at multiple isolated locations at the surface, it means that a few of these regions are seen simultaneously, independently of the NS orientation. This effect is amplified further when the curved path of photons in the NS gravitational field is taken into account. We show the light curves for models 4 and 6 in Figure 8. Despite the factor of two difference in temperature between hot and cold regions, the pulsed fraction is limited to $9 \%-11 \%$, similar to real CCOs.

Overall, the light curves have a few components that correspond to different hot regions. The shape of light curves is 


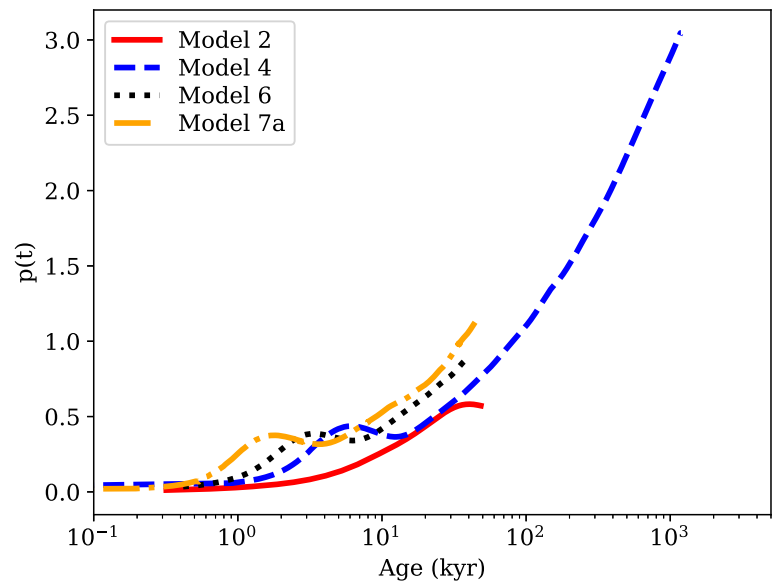

(a)

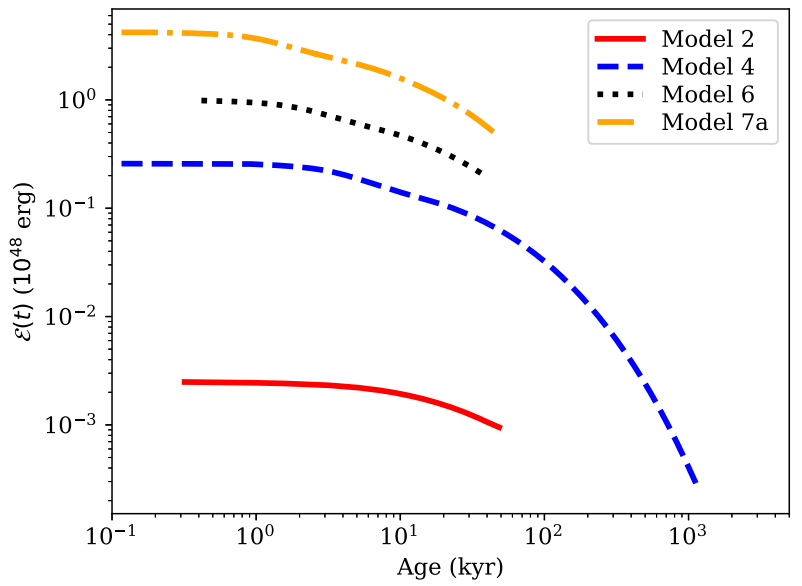

(b)

Figure 5. Evolution of (a) the instantaneous energy exponent Equation (16) and (b) total magnetic energy for different models. Model 7a corresponds to model 7 in Gourgouliatos et al. (2020).

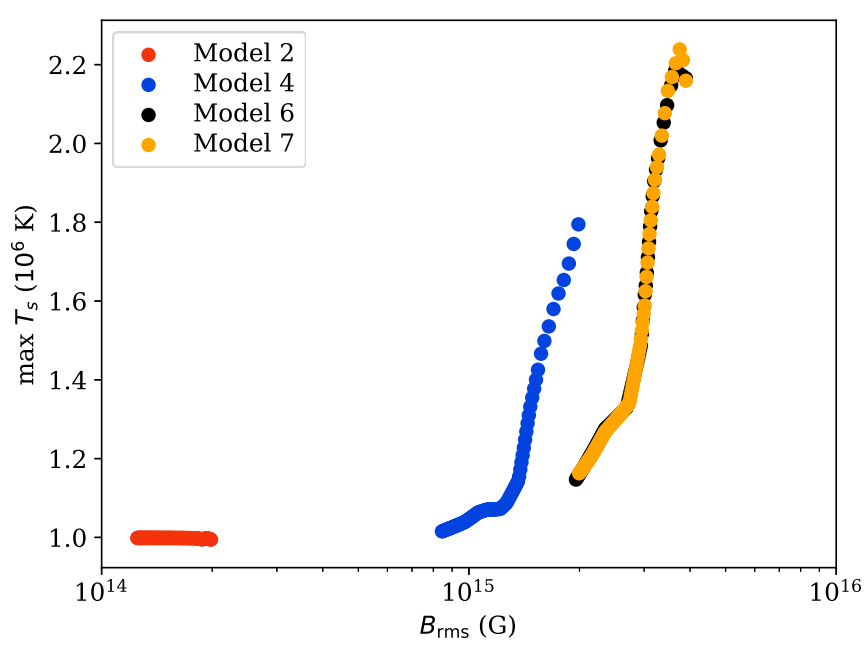

Figure 6. Evolution of maximum surface temperature as a function of $B_{\mathrm{rms}}$.

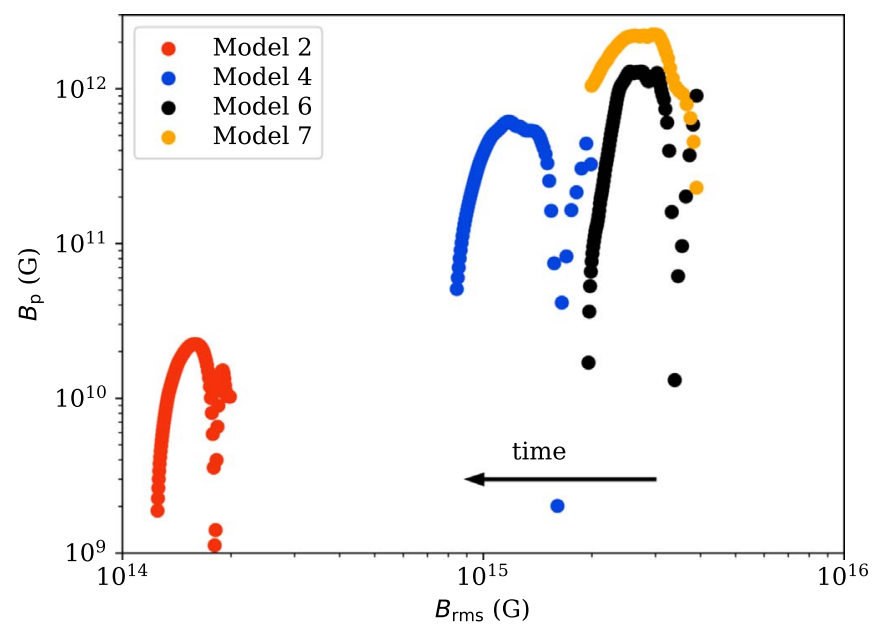

Figure 7. Evolution of dipolar poloidal component of the magnetic field as a function of $B_{\mathrm{rms}}$.

simple because the hot regions are compact. There is no use in discussing how much one light curve peak is higher/lower than another peak, because it is defined by the small-scale structure of the magnetic field. Slightly different configurations of the field could produce a very different light-curve shape.

\section{Discussion}

The main caveats of our simulations are as follows: (1) we do not take NS neutrino cooling into account, (2) we use a simplified treatment of the NS atmosphere, (3) we assume a strong beaming of the thermal photon emission, and (4) no magnetic field evolution in the core is assumed. Below we briefly explain how the addition of these effects could change the results of our simulations.

NS cools down and the core temperature decays below $10^{8} \mathrm{~K}$ assumed in our simulations within the first $10 \mathrm{kyr}$ of the NS life. Therefore, the surface temperatures will be smaller in extended simulations. Nevertheless, the magnetic field isolates certain regions from the core and heats them up, so the temperature difference between hot and cold regions might increase in realistic simulations with NS neutrino core cooling. Another factor that is not taken into account is the neutrino cooling of the crust. It might remove the excess heat from hot spots, limiting their temperatures by some threshold.

In our simulations we assume a temperature dependence between the deep crust and surface in the form of Equation (12). In reality the temperature depends on magnetic fields as well (see, e.g., Potekhin \& Yakovlev 2001). This might change the surface temperature thermal map and could affect the light curves. We assume a strong beaming of the thermal emission proportional to $\cos ^{2} \theta^{\prime}$, which approximately follows the exact numerical calculations of van Adelsberg \& Lai (2006). For weak magnetic fields the beaming might be weaker, which will further decrease the maximum pulsed fraction that we see in our models.

Recently, Gusakov et al. (2020) modeled the ambipolar diffusion in the NS core. They noticed that magnetic field in the core could evolve significantly on timescales of NS cooling, i.e., $10^{4}-10^{6}$ yr (Igoshev \& Popov 2014, 2015, 2020). Such a magnetic field evolution proceeding at the lower boundary condition could noticeably change both the final magnetic field configuration arising as a result of magnetic field evolution in the crust and the surface thermal pattern. 

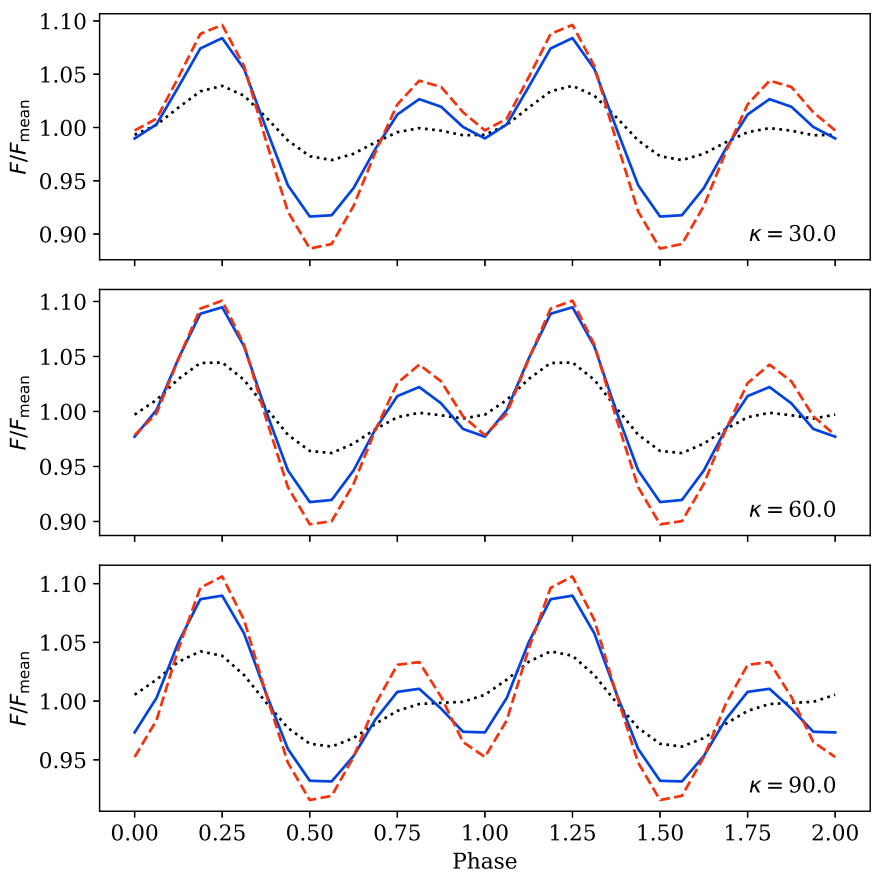

(a)
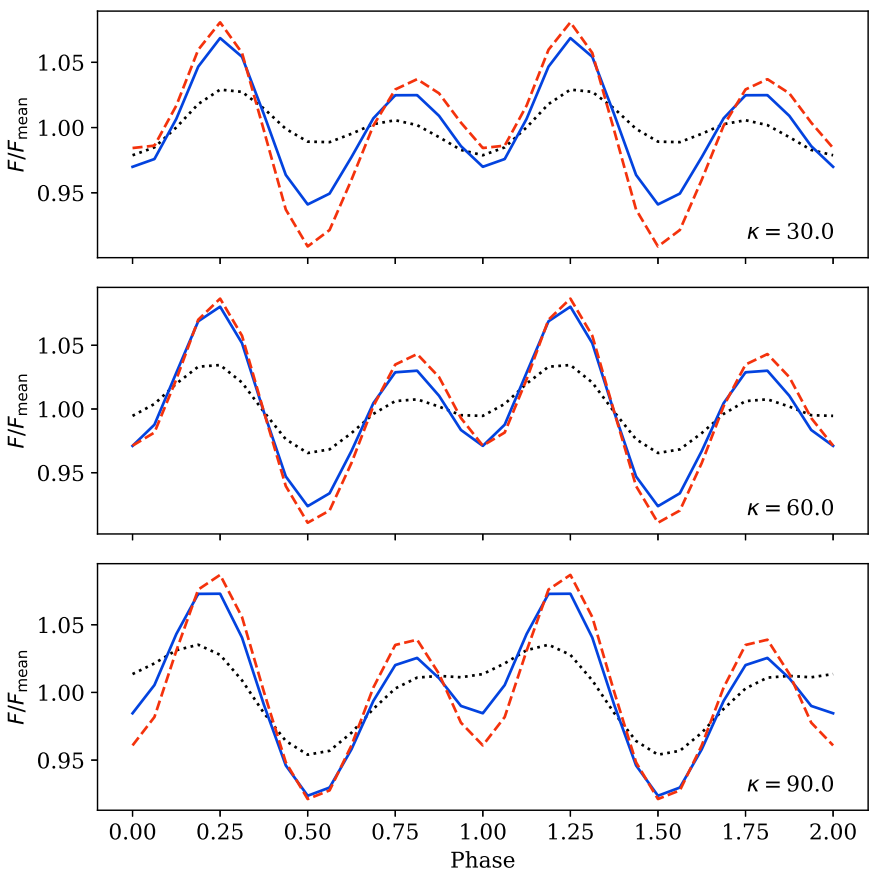

(b)

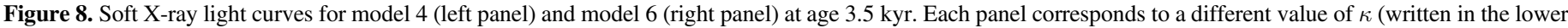
right corner); the black dotted line shows $i=30^{\circ}$, the solid blue line corresponds to $i=60^{\circ}$, and the red dashed line is for $i=90^{\circ}$.

\section{Conclusions}

We perform three-dimensional magnetothermal electronMHD simulations of the NS crust to study the tangled magnetic field configurations. These configurations were suggested to be a possible mechanism to explain the peculiar emission properties of CCOs. We found that these magnetic field configurations lead to the following:

1. Formation of small hot regions with a typical size of $\approx 2 \mathrm{~km}$ that are located at significant separations from each other.

2. The heating correlates with initial total magnetic energy. Most of the crustal heating due to the currents is released during the first $10-20 \mathrm{kyr}$ of NS evolution. The power released owing to the magnetic field decay could reach values of $3 \times 10^{35} \mathrm{erg} \mathrm{s}^{-1}$. Only a fraction of this power is emitted as thermal $\mathrm{X}$-ray radiation from the NS surface.

3. In our simulations with initial total magnetic energy of $E_{\mathrm{tot}, 0}=2.5 \times 10^{47} \mathrm{erg}$, the hot regions have temperatures 1.7 times larger than the bulk surface temperature of NS. In our simulations with $E_{\mathrm{tot}, 0}=10^{48} \mathrm{erg}$, the hot regions have temperatures that are 2.2 times larger than the bulk surface temperature. These factors are compatible with ones seen in the X-ray observations of CCOs.

4. The maximum surface temperature decays exponentially on a timescale of $\approx 15 \mathrm{kyr}$. The maximum temperature approaches the bulk surface temperature already after $\approx 20 \mathrm{kyr}$.

5. The resulting light curves show modulations with maximum PFs of $9 \%-11 \%$ in the case of models 4,5 , 6, and 7. These small PFs can be explained by the fact that hot regions are located at multiple positions on the NS, and it is impossible to choose such an orientation where none are present. This is a reason why larger $E_{\mathrm{tot}, 0}$ does not necessarily lead to an increase in PF. Regions become hotter, but a few of them are still seen simultaneously.

6. The final poloidal magnetic field correlates with rms magnetic field.

Overall, the hidden magnetic field could provide enough energy to explain enhanced thermal emission of CCOs. A part of the tangled magnetic field energy is released through thermal emission from the NS surface via emission of small hot spots. The small size of these spots is related to a typical size of magnetic field loops assumed as the initial condition in our simulations. These sizes are comparable to the NS crust depth and could be produced in a stochastic dynamo. Therefore, the main difference between CCOs and magnetars in this scenario is the typical size of the magnetic field. In magnetars the magnetic field is large scale, while in CCOs it is mostly small scale.

This work was supported by STFC grant ST/S000275/1. This work was undertaken on ARC3 and ARC4, part of the High Performance Computing facilities at the University of Leeds, UK.

\section{ORCID iDs}

Andrei P. Igoshev (i) https://orcid.org/0000-0003-2145-1022 Konstantinos N. Gourgouliatos (1) https://orcid.org/00000002-1659-1250

Rainer Hollerbach (1) https://orcid.org/0000-0001-8639-0967 Toby S. Wood (1) https://orcid.org/0000-0003-1044-170X

\section{References}

Beloborodov, A. M. 2002, ApJL, 566, L85

Bernal, C. G., Lee, W. H., \& Page, D. 2010, RMxAA, 46, 309

Bernal, C. G., Page, D., \& Lee, W. H. 2013, ApJ, 770, 106

Blandford, R. D., Applegate, J. H., \& Hernquist, L. 1983, MNRAS, 204, 1025 
Bogdanov, S., Ng, C. Y., \& Kaspi, V. M. 2014, ApJL, 792, L36

Brandenburg, A. 2020, ApJ, 901, 18

Brandenburg, A., Johansen, A., Bourdin, P. A., et al. 2020, arXiv:2009.08231

Chandrasekhar, S. 1950, Radiative Transfer (Oxford: Clarendon Press)

Chevalier, R. A. 1989, ApJ, 346, 847

DeDeo, S., Psaltis, D., \& Narayan, R. 2001, ApJ, 559, 346

De Grandis, D., Turolla, R., Wood, T. S., et al. 2020, ApJ, 903, 40

De Luca, A. 2008, in AIP Conf. Ser. 983, 40 Years of Pulsars: Millisecond

Pulsars, Magnetars and More, ed. C. Bassa et al. (Melville, NY: AIP), 311

De Luca, A. 2017, JPhCS, 932, 012006

Dormy, E., Cardin, P., \& Jault, D. 1998, E\&PSL, 160, 15

Gaensler, B. M., \& Slane, P. O. 2006, ARA\&A, 44, 17

Goldreich, P., \& Reisenegger, A. 1992, ApJ, 395, 250

Gotthelf, E. V., \& Halpern, J. P. 2007, ApJL, 664, L35

Gotthelf, E. V., \& Halpern, J. P. 2009, ApJL, 695, L35

Gotthelf, E. V., Halpern, J. P., \& Alford, J. 2013, ApJ, 765, 58

Gotthelf, E. V., Halpern, J. P., \& Seward, F. D. 2005, ApJ, 627, 390

Gourgouliatos, K. N., \& Cumming, A. 2014, MNRAS, 438, 1618

Gourgouliatos, K. N., Hollerbach, R., \& Igoshev, A. P. 2020, MNRAS, 495,1692

Gourgouliatos, K. N., Wood, T. S., \& Hollerbach, R. 2016, PNAS, 113, 3944

Gudmundsson, E. H., Pethick, C. J., \& Epstein, R. I. 1982, ApJL, 259, L19

Gudmundsson, E. H., Pethick, C. J., \& Epstein, R. I. 1983, ApJ, 272, 286

Gusakov, M. E., Kantor, E. M., \& Ofengeim, D. D. 2020, MNRAS, 499, 4561

Halpern, J. P., \& Gotthelf, E. V. 2010, ApJ, 709, 436

Ho, W. C. G. 2011, MNRAS, 414, 2567

Hollerbach, R., \& Rüdiger, G. 2004, MNRAS, 347, 1273

Igoshev, A. P., Elfritz, J. G., \& Popov, S. B. 2016, MNRAS, 462, 3689

Igoshev, A. P., Hollerbach, R., Wood, T., \& Gourgouliatos, K. N. 2021, NatAs, 5,145

Igoshev, A. P., \& Popov, S. B. 2014, MNRAS, 444, 1066
Igoshev, A. P., \& Popov, S. B. 2015, AN, 336, 831

Igoshev, A. P., \& Popov, S. B. 2020, MNRAS, 499, 2826

Igoshev, A. P., Popov, S. B., \& Turolla, R. 2014, AN, 335, 262

Kaspi, V. M. 2010, PNAS, 107, 7147

Kaspi, V. M., \& Beloborodov, A. M. 2017, ARA\&A, 55, 261

Keane, E. F., \& Kramer, M. 2008, MNRAS, 391, 2009

Lorimer, D. R., \& Kramer, M. 2004, Handbook of Pulsar Astronomy, Vol. 4 (Cambridge: Cambridge Univ. Press)

Luo, J., Ng, C. Y., Ho, W. C. G., et al. 2015, ApJ, 808, 130

Nagakura, H., Burrows, A., Radice, D., \& Vartanyan, D. 2020, MNRAS, 492, 5764

Page, D., Lattimer, J. M., Prakash, M., \& Steiner, A. W. 2004, ApJS, 155, 623

Pavlov, G. G., Sanwal, D., \& Teter, M. A. 2004, in IAU Symp. 218, Young Neutron Stars and Their Environments, ed. F. Camilo \& B. M. Gaensler (San Francisco, CA: Astronomical Society of the Pacific), 239, arXiv:astro$\mathrm{ph} / 0311526$

Philippov, A., Timokhin, A., \& Spitkovsky, A. 2020, PhRvL, 124, 245101

Pons, J. A., Reddy, S., Prakash, M., Lattimer, J. M., \& Miralles, J. A. 1999, ApJ, 513, 780

Potekhin, A. Y., \& Yakovlev, D. G. 2001, A\&A, 374, 213

Raynaud, R., Guilet, J., Janka, H.-T., \& Gastine, T. 2020, SciA, 6, eaay2732

Rea, N., Borghese, A., Esposito, P., et al. 2016, ApJL, 828, L13

Thompson, C., \& Murray, N. 2001, ApJ, 560, 339

Turolla, R. 2009, in Isolated Neutron Stars: The Challenge of Simplicity, ed. W. Becker, Vol. 357 (Berlin: Springer), 141

van Adelsberg, M., \& Lai, D. 2006, MNRAS, 373, 1495

Viganò, D., \& Pons, J. A. 2012, MNRAS, 425, 2487

Wareing, C. J., \& Hollerbach, R. 2009, PhPl, 16, 042307

Wareing, C. J., \& Hollerbach, R. 2010, JPIPh, 76, 117

Wood, T. S., \& Hollerbach, R. 2015, PhRvL, 114, 191101

Zavlin, V. E., Pavlov, G. G., Sanwal, D., \& Trümper, J. 2000, ApJL, 540, L25 